\title{
Disorder-induced localisation and suppression of superconductivity in $\mathrm{YSr}_{2} \mathrm{Cu}_{3} \mathrm{O}_{6+x}$
}

\author{
Andrea Gauzzi \\ IMPMC, Sorbonne Université, UMR7590 CNRS, IRD, MNHN, 4, place Jussieu, \\ 75005 Paris, France \\ E-mail: andrea.gauzzi@sorbonne-universite.fr
}

Gianni Profeta, Alessandra Continenza

Dipartimento di Scienze Fisiche e Chimiche, Università dell'Aquila, 67010 Coppito, Italy

\author{
Fabio Bernardini, Sandro Massidda \\ Dipartimento di Fisica, Università di Cagliari, 09042 Monserrato, Italy
}

\begin{abstract}
.
By means of $a b$ initio calculations within the local density approximation to density functional theory, we investigate the electronic structure of the $60 \mathrm{~K}$ superconductor $\mathrm{YSr}_{2} \mathrm{Cu}_{3} \mathrm{O}_{6+x}$ (YSCO). We focus on the effects of the $\mathrm{Sr} / \mathrm{Ba}$ substitution and on the main structural modifications induced by this substitution experimentally found in the Sr compound, namely the tetragonal symmetry and the oxygen disorder in the basal plane. In the calculations, this disorder is simulated by using a supercell approach. Due to band structure effects, we find a larger stabilisation free energy of the orthorhombic structure in $\mathrm{YBa}_{2} \mathrm{Cu}_{3} \mathrm{O}_{6+x}$ (YBCO). In YSCO, the tetragonal disordered phase is found to be stabilized by oxygen overdoping $(x>1)$ and by sufficiently large massenhancement factors, $\gamma>5$. The analysis of the atomic site projected density of states suggests that oxygen disorder in the $\mathrm{CuO}$ basal planes of YSCO induces hole localisation, which accounts for the large $30 \mathrm{~K}$ reduction of $T_{c}$ with respect to YBCO.
\end{abstract}

Keywords: superconductivity, first-principles calculations, charge localisation

\section{Introduction: role of oxygen disorder on superconductivity in cuprates}

More than thirty years after the discovery of high-temperature superconductivity (HTSC) in cuprates [1], the explanation of this extraordinary phenomenon remains one of the great challenges of condensed matter physics. One difficulty is the complex crystal structure combined with chemical disorder, two characteristic features of these superconductors. Typically, this disorder is caused by oxygen doping in the pristine insulating phase, which is required to create mobile holes in the $\mathrm{CuO}_{2}$ planes and thus to induce superconductivity. It is therefore important to investigate the effects of oxygen 
disorder on the electronic properties, considering that the local crystal structure may differ from the average one probed by diffraction techniques.

In the present work, we address this issue in the case of $\mathrm{YSr}_{2} \mathrm{Cu}_{3} \mathrm{O}_{6+x}$ (YSCO) $[2,3,4,5]$, the counterpart of the well-known $\mathrm{YBa}_{2} \mathrm{Cu}_{3} \mathrm{O}_{6+x}$ (YBCO) compound where $\mathrm{Ba}$ is fully substituted by Sr. Contrary to the expectation that the chemical pressure exerted by the comparatively small $\mathrm{Sr}$ ion should enhance superconductivity, in YSCO the critical temperature, $T_{c}$, is unexpectedly reduced from $90 \mathrm{~K}$ to $60 \mathrm{~K}$. This result is surprising considering that the above substitution does not perturb the superconducting $\mathrm{CuO}_{2}$ planes and successfully induces the desired chemical pressure effect [4]. Here, we consider the possibility that the above reduction is due to the disorder of the oxygen atoms in the basal planes in superconducting $\mathrm{YSr}_{2} \mathrm{Cu}_{3} \mathrm{O}_{6+x}$ for $x \approx 1[4,5,6,7]$, which contrasts the configuration of well-ordered $\mathrm{CuO}$ chains in optimally doped $\mathrm{YBa}_{2} \mathrm{Cu}_{3} \mathrm{O}_{6+x}$ for an almost identical oxygen stoichiometry, $x \approx 0.9$. As a result, the crystal structure of YSCO is tetragonal $P 4 / \mathrm{mmm}$, while the crystal structure of optimally doped YBCO is orthorhombic Pmmm, which is expected to alter significantly the electronic structure, as suggested by previous experimental [8], numerical [9] and theoretical [10] studies on the effect of local oxygen ordering in YBCO.

In order to investigate the changes in the electronic structure caused by both the $\mathrm{Sr} / \mathrm{Ba}$ substitution and the oxygen disorder, we perform the first $a b$ initio band structure calculations for the fully doped structure (i.e. with $x=1$ ) of YSCO, both in the ordered and in the disordered cases. Our results show that in YBCO the absence of $\mathrm{CuO}$ chains arising from this disorder strongly reduces the density of states (DOS) at the Fermi level $E_{F}$, and decreases the doping of the superconducting $\mathrm{CuO}_{2}$ planes. Due to structural and chemical effects, we find that the same is not true for YSCO.

\section{Numerical methods}

For the calculations, we use the 'all-electron' full-potential linearised-augmented-planewaves (FLAPW) method in the local density approximation (LDA) to the density functional theory, as described elsewhere $[11,12]$. In the interstitial region we use plane waves with wave vector up to $k_{\max }=3.8$ a.u. and muffin-tin radii: $R_{Y}=2.4$ a.u., $R_{C u}=$ 1.95 a.u., $R_{S r}=2.8$ a.u., and $R_{O}=1.6$ and 1.49 a.u. for the in-plane and apical oxygens, respectively. Inside the muffin-tin spheres, angular momenta up to $l_{\max }=8$ are used to expand potential, charge density and wave functions. The Brillouin zone is sampled using the special $k$-point technique of the Monkhorst-Pack scheme [13] and also the linear tetrahedron method with up to $125 k$-points in the irreducible Brillouin zone to calculate the density of states. The structural parameters of the ordered and disordered YBCO and YSCO equilibrium structures and the relative differences in total energy are computed in the density-functional-theory pseudopotential plane-wave framework. For

this calculation we use the VASP code $[14,15]$ based on ultrasoft pseudopotentials for all the elements involved [16]; Y, Sr and $\mathrm{Ba} p$-semicore states are included in the valence. To treat the exchange-correlation energy functional, we use LDA in the Ceperley-Alder 
[17] form parametrised by Perdew and Zunger [18]. We find that LDA gives satisfactory agreement with the experimental (and GGA [19] results, within 1-2\%), as shown in Table 1 below. A kinetic energy cutoff of $36 \mathrm{Ry}$ is found to be sufficient to achieve convergence of the electronic and structural parameters. Brillouin zone integration of the formula-unit reciprocal cell is performed on a $8 \times 8 \times 4$ Monkhorst-Pack mesh [13]. To ensure energy convergence with respect to $k$-point sampling, we use an electronic finitetemperature technique with a $0.1 \mathrm{eV}$ Gaussian broadening of the occupation numbers. Atomic positions, cell size and shape are optimised by computing the stresses and the forces with a quasi-Newton algorithm. Convergence of the total energy within $1 \mathrm{meV}$ is achieved by varying the structural parameters.

\section{Numerical results}

Hereafter, we shall adopt the notation used in Ref. [20] to label the distinct $\mathrm{Cu}$ and O sites in the orthorhombic Pmmm symmetry (see Fig. 1), i.e. Cu1 for the copper in the basal plane at $(0,0,0), \mathrm{Cu} 2$ for the copper in the $\mathrm{CuO}_{2}$ plane at $(0,0, z), \mathrm{O} 1$ for the apical oxygen at $(0,0, z), \mathrm{O} 2$ and $\mathrm{O} 3$ for the oxygens in the $\mathrm{CuO}_{2}$ plane at $(1 / 2,0, z)$ and $(0,1 / 2, z)$, respectively, $\mathrm{O} 4$ for the chain oxygen at $(0,1 / 2,0)$ and $\mathrm{O} 5$ for the oxygen in the basal plane at $(1 / 2,0,0)$. Before presenting the results of the calculations, we recall the main features of the band structure of YBCO [21]. There are four partially filled bands at $E_{F}$. Two of them arise from the hybridisation of the Cu2(3d)-O2(p)-O3(p) orbitals in the superconducting $\mathrm{CuO}_{2}$ planes and have two-dimensional character. Their dispersion is strong and nearly symmetric along X-S and Y-S. The other two bands arise from the hybridisation of the $\mathrm{Cu} 1(3 \mathrm{~d})-\mathrm{O} 1(\mathrm{p})-\mathrm{O} 4(\mathrm{p})$ orbitals in the basal $\mathrm{CuO}$ plane and are therefore associated with the $\mathrm{CuO}$ chains. The character of these bands is onedimensional; they are both anti-bonding with respectively $\sigma$ and $\pi$ character.

The first issue we address is the purely chemical effect of the isovalent $\mathrm{Ba} / \mathrm{Sr}$ substitution. To do this, we calculate the band structure of YSCO in the 'frozen' YBCO structure and compare it with that of YBCO (see Figure 2). The close similarity between the two band structures near $E_{F}$ indicates that the effect is small, but not negligible. The only relevant difference concerns the chain-related $\pi$ bands (in particular the apical $\mathrm{O} p_{x}$ and $\mathrm{Cu} d_{x z}$ states just below $E_{F}$ at $S$ ), which are located at higher energy in YBCO. In conclusion, the larger effect of the Sr substitution is steric, as expected.

We now attempt to understand why in YSCO nature favors the average tetragonal symmetry arising from oxygen disorder in the $\mathrm{CuO}$ plane of the orthorhombic symmetry of YBCO. We first minimise the total energy $E$ to determine the unit cell parameters $a$ and $b$ at equilibrium of the hypothetical orthorhombic structure of YSCO with $x=1$. From Table 1 we see that both the average $\frac{a+b}{2}$ and $c$ are in good agreement with the experimental parameters within less than $\approx 2 \%$. To evaluate the reliability of the method, the same calculations are carried out for YBCO as well. From Table 1 we note the same trends and a satisfactory agreement between calculated and experimental parameters for this compound as well. The orthorhombic distortion, expressed by the 

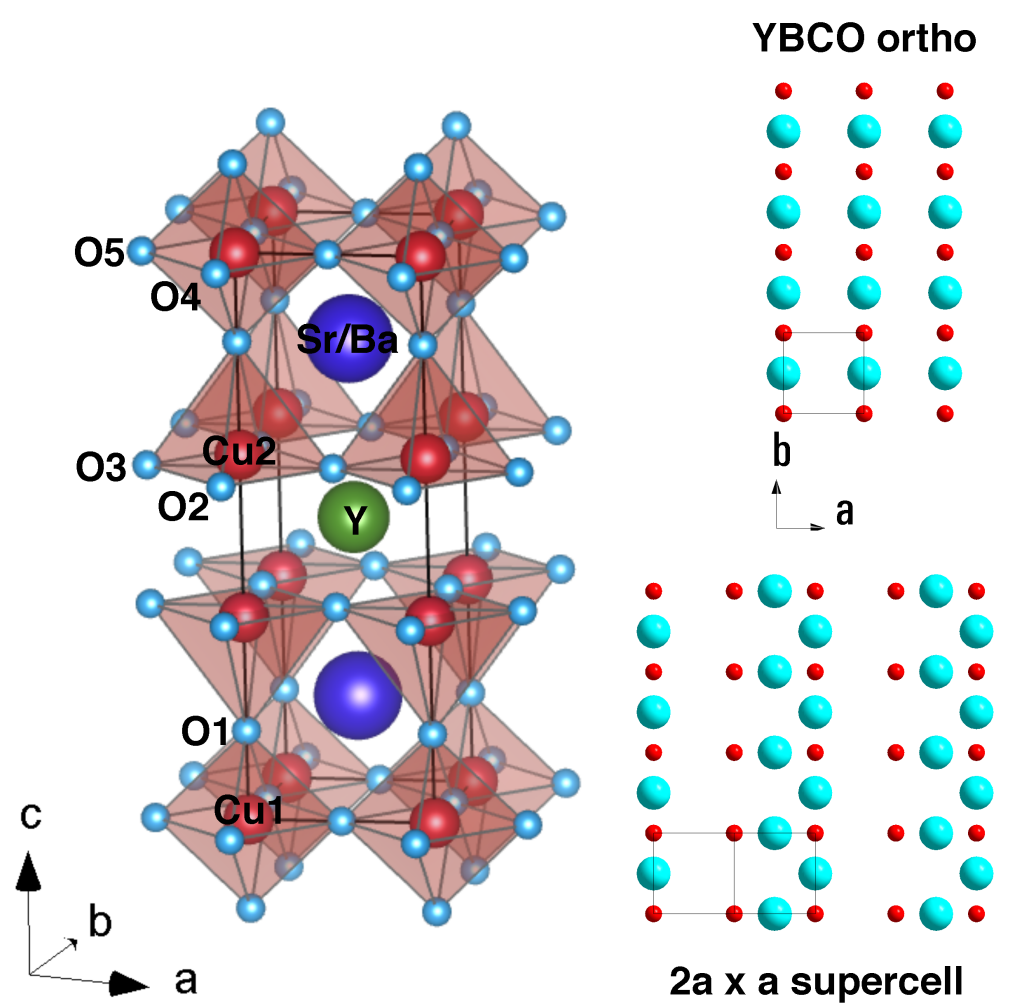

YSCO tetra

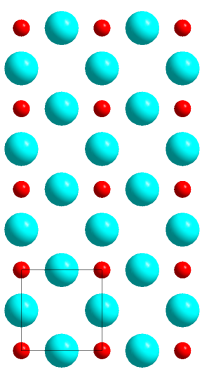

Figure 1. Left: crystal structure of YBCO and of YSCO for $x=1$. Red and light-blue spheres indicate copper and oxygen atoms, respectively. The solid black line indicates the unit cell. The labels of non equivalent $\mathrm{Cu}$ and $\mathrm{O}$ sites are as described in the text and in Ref. [20]. Note that, in the tetragonal $P 4 / \mathrm{mmm}$ structure of YSCO, the $\mathrm{O} 4$ and $\mathrm{O} 5$ oxygen sites are partially occupied at 50\%, while, in the orthogonal Pmmm structure of YBCO, these two sites are fully occupied and empty, respectively. Right: the following oxygen ordering schemes in the $\mathrm{CuO}$ basal planes are shown: (i) orthorhombic $\mathrm{YBCO}$ with ordered $\mathrm{CuO}$ chains along $b$; (ii) tetragonal $\mathrm{YSCO}$ with equal occupancy of the $\mathrm{O} 4$ and $\mathrm{O} 5$ sites along the $a$ - and $b$-directions; (iii) $2 a \times a$ and (iv) $\sqrt{2} a \times \sqrt{2} a$ supercells used in the $a b$ initio calculations to simulate the absence of $\mathrm{CuO}$ chains in the previous case (ii) of tetragonal YSCO.

$b / a$ ratio, is larger in YSCO. Indeed, the smaller Sr cation is more effective in reducing the $a$ parameter, whilst the $b$ parameter is mainly determined by the Cu1-O4 distance.

To investigate how structural relaxation affects the electronic structure of YSCO, we calculate the band structure of the hypothetical orthorhombic structure (with $\mathrm{CuO}$ chains), reported in Table 1. The result is shown in Figure 2. We notice the different shape of the chain-related $\pi$-antibonding bands, located near $E_{F}$ in YBCO and entirely occupied in YSCO. At the $S$-point, these bands are found at about $0.5 \mathrm{eV}$ below $E_{F}$. As a result, the density of states at $E_{F}$ is reduced to about half of that of YBCO. However, the position of the $\mathrm{CuO}_{2} d-p_{\sigma}$ Fermi level crossings is nearly unchanged, implying very similar band fillings in the ordered orthorhombic phase of both compounds.

The point that remains to be studied is how disorder affects the electronic properties 

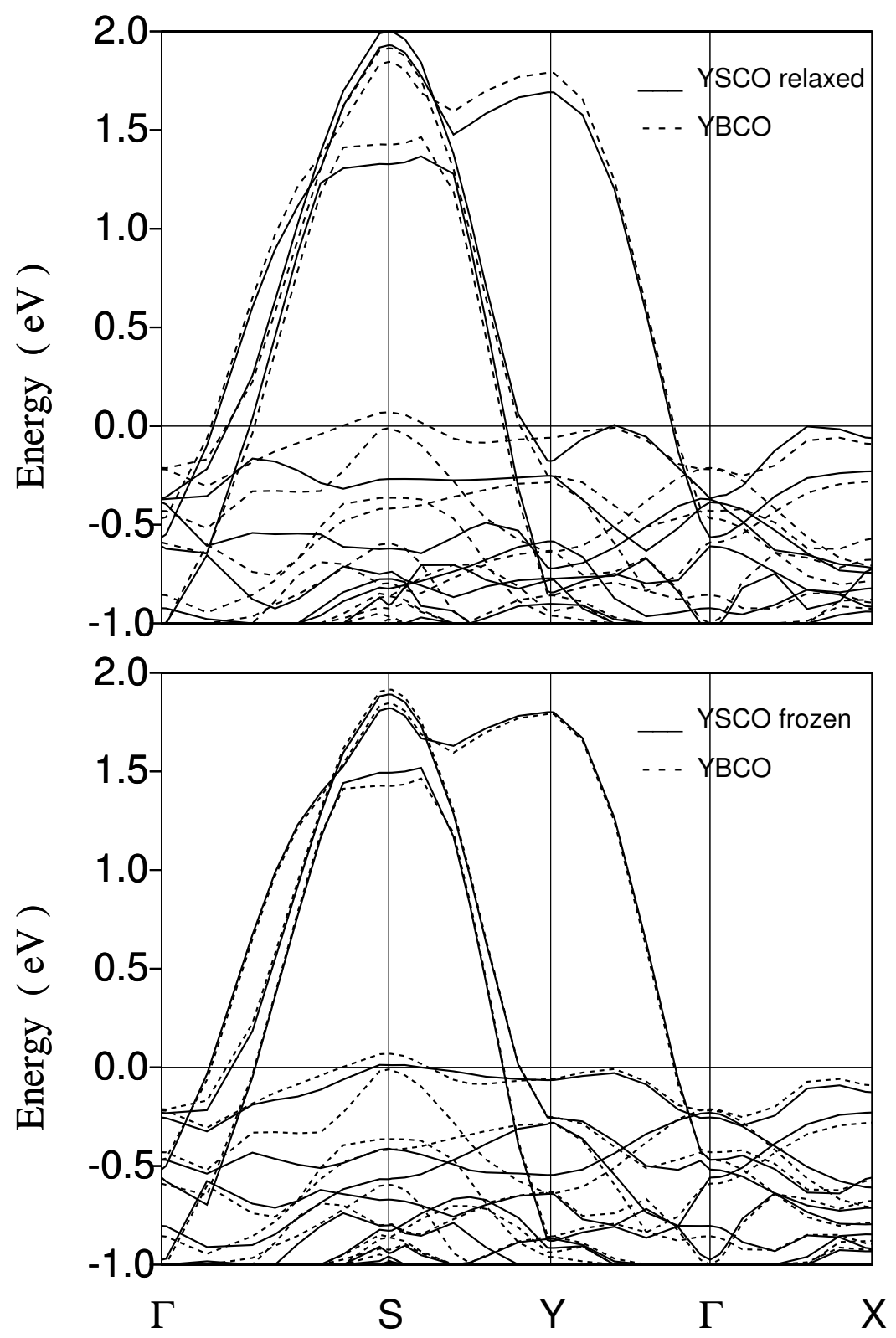

Figure 2. Lower panel: band structures of YSCO (solid lines) and of YBCO (dashed lines) calculated in both cases using the 'frozen' crystal structure of the latter compound. Upper panel: the same as above using for YSCO the relaxed structure obtained by minimising the total energy. 
Table 1. Comparison between experimental and calculated unit cell parameters for ordered and disordered $\mathrm{YSr}_{2} \mathrm{Cu}_{3} \mathrm{O}_{6+x}$ (YSCO) and $\mathrm{YBa}_{2} \mathrm{Cu}_{3} \mathrm{O}_{6+x}$ (YBCO) in the orthorhombic structure where the $\mathrm{CuO}$ chains run along the $b$-direction. The experimental values labeled as ${ }^{*}$ and ${ }^{\dagger}$ are taken from Refs. [4] and [22], respectively.

\begin{tabular}{ccccccc}
\hline \hline \multicolumn{3}{c}{ YSCO } & \multicolumn{2}{c}{ YBCO } \\
\hline & Exp. $^{*}(x=1.3)$ & Calc. $(x=1)$ & Exp. $^{\dagger}(x=0.9)$ & Calc. $(x=1)$ \\
\hline & & ord. & dis. & & ord. & dis. \\
\hline \hline $\mathbf{a}[\AA]$ & $3.79167(15)$ & 3.6596 & 3.7677 & $3.8166(3)$ & 3.8057 & 3.8710 \\
\hline $\mathbf{b}[\AA]$ & $3.79167(15)$ & 3.8025 & 3.7362 & $3.8852(3)$ & 3.8604 & 3.8334 \\
\hline $\mathbf{c}[\AA]$ & $11.4075(11)$ & 11.1732 & 11.1368 & $11.6901(9)$ & 11.4642 & 11.4484 \\
\hline $\mathbf{b} / \mathbf{a}$ & 1 & 1.039 & 0.991 & 1.0168 & 1.0143 & 0.990 \\
\hline $\mathbf{c} / \mathbf{a}$ & 3.0078 & 3.053 & 2.955 & 3.0554 & 3.0123 & 2.9574 \\
\hline \hline
\end{tabular}

of YSCO. To simulate the oxygen disorder leading to the absence of chains, we assume a realistic local arrangement of the oxygens compatible with the average tetragonal symmetry found experimentally. NQR data [7] give evidence of a random distribution of 1.3 oxygens per unit cell in the two equivalent $\mathrm{O} 4(0,1 / 2,0)$ and $\mathrm{O} 5(1 / 2,0,0)$ positions of the tetragonal symmetry (see Figure 1). To take this random distribution into account with a reasonable computational effort, we consider two different supercells in the $a b$ plane schematically shown in Figure 1. In both cases the oxygens are ordered in such a way as to avoid almost completely the formation of $\mathrm{CuO}$ chains, thus simulating the chain disorder. The first supercell is a primitive orthorhombic, $2 a \times a$, with one of the two oxygen atoms in the $\mathrm{O} 4$ site in the $\mathrm{CuO}$ basal plane shifted to the $\mathrm{O} 5$ position. A zig-zag of $\mathrm{O}$ atoms is therefore formed along the $b$ axes and one out of two chains is missing. The second supercell is a tetragonal $\sqrt{2} a \times \sqrt{2} a$ (rotated by $45^{\circ}$ ) with the oxygens ordered along the diagonal $a b$-direction. No significant orbital overlap (and hence no band formation) is expected to arise from this arrangement, being the O-O distance along this direction as large as $2.68 \AA$.

For both supercells, we calculate the band structure using the theoretical equilibrium lattice parameters obtained in the pseudopotential approach and reported in Table 1. We recall that these parameters are close to the experimental ones $[4,7]$. Since the calculations on both supercells lead to physically similar conclusions, in the following we discuss only the case of the first supercell, for the Brillouin zone folding is simpler in this case. Namely, the single-cell $X$-point folds into the supercell $\Gamma^{\prime}$-point, while both $Y$ and $S$ of the single cell fold into $Y^{\prime}$ of the supercell. The $X^{\prime}$ of the supercell corresponds to the $\Gamma-X$ midpoint, where the folding occurs.

In Figure 3 we compare the supercell result with that of the ordered single-cell phase (i.e. with $\mathrm{CuO}$ chains) of Figure 2. To do this, we report the single-cell result of Figure 2 folded into the supercell Brillouin zone. We note that the shape of the bands associated with the $\mathrm{CuO}_{2}$ planes are nearly unchanged by the disorder in 
both compounds, as expected since the disorder is located far apart from these planes. Specifically, in $\mathrm{YBa}_{2} \mathrm{Cu}_{3} \mathrm{O}_{6+x}$ these bands are nearly rigidly shifted downward in energy in the disordered phase (see, in particular, the region around the $\Gamma$ and $Y^{\prime}$ points). This indicates that the disorder leads to a larger filling of the $d-p_{\sigma}$ bands in YBCO. On the other hand, in $\mathrm{YSr}_{2} \mathrm{Cu}_{3} \mathrm{O}_{6+x}$ the disorder does not affect significantly the filling of these bands. A similar situation occurs in $\mathrm{YSr}_{2} \mathrm{Cu}_{3} \mathrm{O}_{6+x}$ in the case of the ordered single-cell shown in Figure 2, where the structural relaxation does not appreciably change the $d-p_{\sigma}$ crossings.

We now focus on the $d_{z^{2}-y^{2}} \sigma$-bands associated with the $\mathrm{CuO}$ chains. From Figure 3 we see for both compounds a strong suppression of the dispersion of the bands. One of these, located at energies $\approx 0.5 \mathrm{eV}$ above $E_{F}$ and crossing the Fermi level close to the S'-point in YSCO, is nearly flat. The second band is located around $1.2 \mathrm{eV}$ above $E_{F}$, at energies $\approx 0.8 \mathrm{eV}$ lower than in the ordered case. The lowering of the empty antibonding bands of the chains (that is even larger in the second supercell, not shown in any figure) implies a less effective binding (lower antibonding band) in the disordered structure, as discussed in detail below. Similar features are found in both compounds by taking into account the oxygen disorder. Nevertheless, in YSCO, unlike in YBCO, a band crosses $E_{F}$ in the disordered phase near $S^{\prime}$, while it does not in the ordered phase. Thanks to such disorder-induced band crossing, a hole transfer from the $d_{z^{2}-y^{2}}$ $\sigma$-band to the $d-p_{\sigma}$ bands of the superconducting $\mathrm{CuO}_{2}$ planes is enabled. This tends to compensate the opposite effect of hole depletion induced by the flat $d-p_{\pi}$ bands.

The above disorder-induced alterations of the electronic structure are observed also in the atomic site projected partial density of states (PDOS) of the first supercell (see Figure 4). The PDOS in the ordered and disordered structures shows sizable differences only for the Cu1, O1 and O4 sites in the basal planes. Namely, the Cu1 and O4 PDOS of the disordered structure reveals a poor hybridisation between $\mathrm{O}(\mathrm{p})$ and $\mathrm{Cu}(3 \mathrm{~d})$ orbitals, as also shown by a pronounced peak appearing in the $\mathrm{O} 4 \mathrm{PDOS}$ at $\simeq-5 \mathrm{eV}$, a signature of non-bonding state. This is our main result, which suggests charge localisation in the basal planes. As a result, hole transfer from these planes to the $\mathrm{CuO}_{2}$ planes, the mechanism enabling the metallic (and superconducting) state, is hindered. This scenario is consistent with an earlier model proposed by Burdett [10]. Interestingly, the PDOS result for the $\mathrm{Cu} 2, \mathrm{O} 2$ and $\mathrm{O} 3$ sites suggests that the $\mathrm{CuO}_{2}$ planes are marginally affected by the fragmentation of the $\mathrm{CuO}$ chains.

\section{Discussion}

We try to understand why oxygen disorder is favored in YSCO by computing the free energy $F$ in the ordered and in the disordered phase of both YSCO and YBCO. We first compare the total energy $E$ contributions. As before, the disorder is simulated by the previous two supercells. Our calculations indicate that the differences of total energy between disordered and ordered structures are similar in YBCO and in YSCO. For both compounds, we obtain a difference $\Delta E \approx 0.5 \mathrm{eV} /$ supercell in excellent agreement 

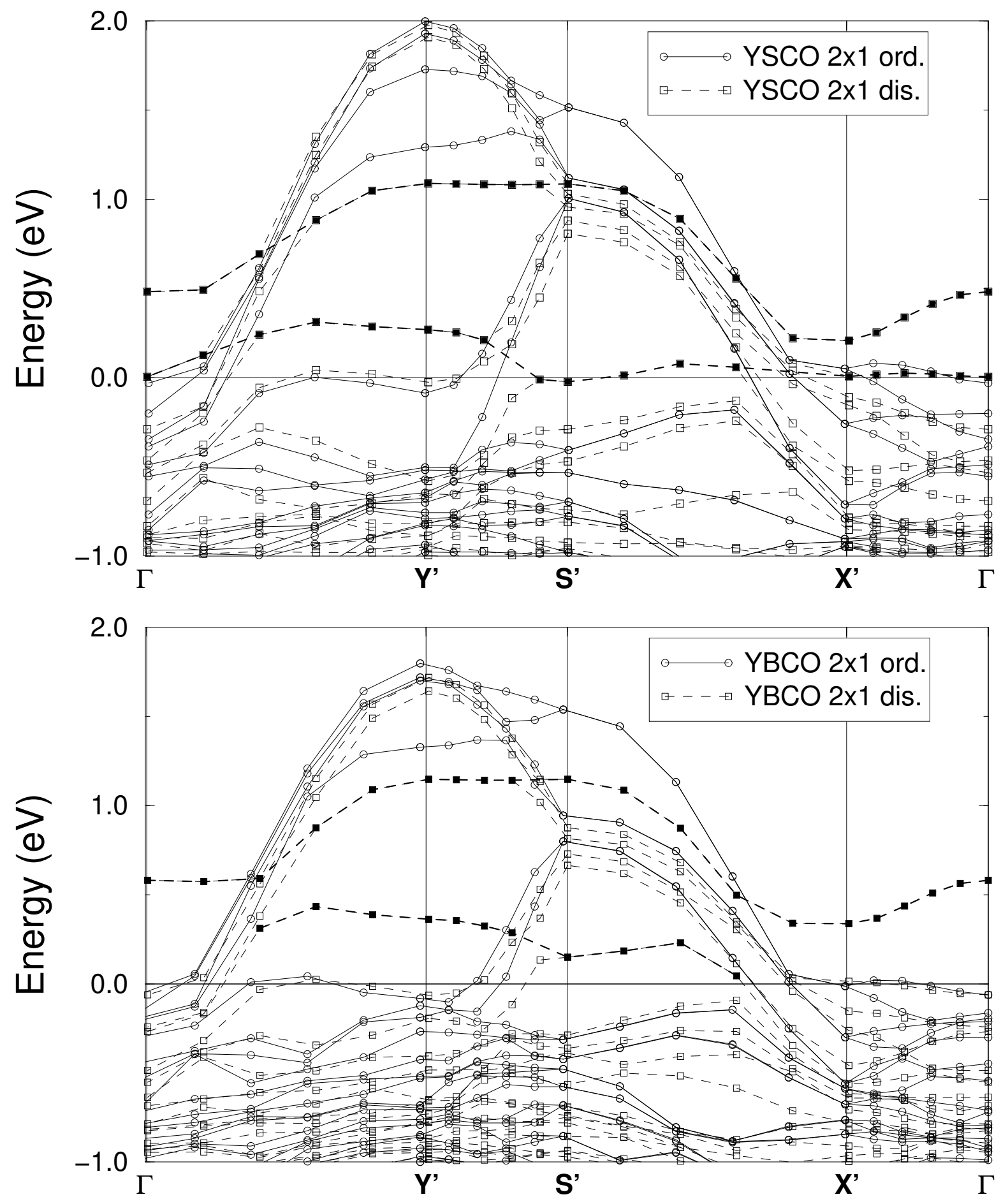

Figure 3. Band structures of YSCO and YBCO calculated for the ordered and disordered $(2 a \times a)$ structures of Figure 1. Black dashed lines with full symbols highlight bands related to $d_{z^{2}-y^{2}}-\sigma$ bands coming from $\mathrm{Cu}-\mathrm{O}$ chains. As no symmetry analysis has been included, lines are only a guide to the eye. 

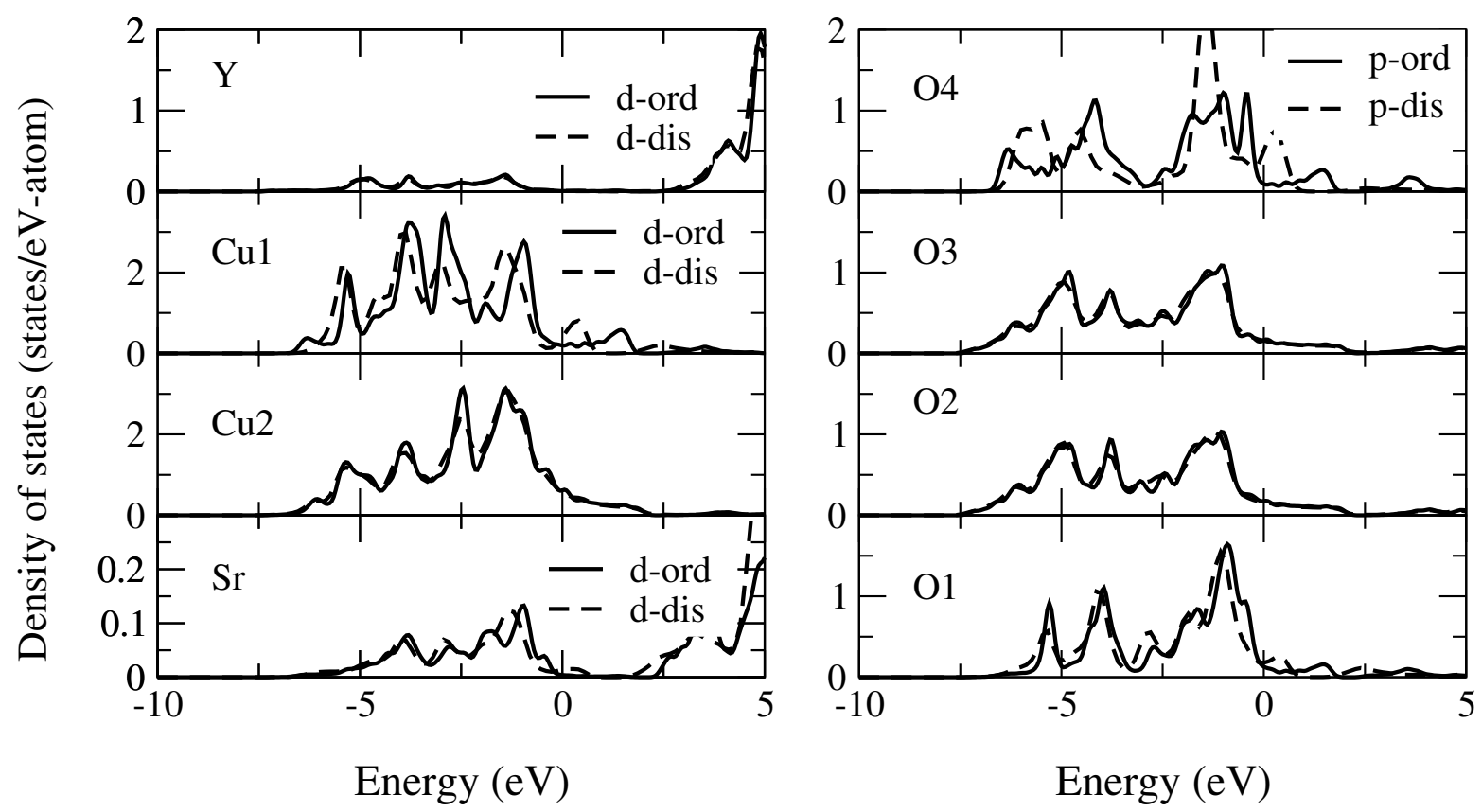

Figure 4. Projected density of states (PDOS) of the YSCO ordered and disordered $(2 a \times a)$ supercell structures of Figure 1. Site labels are defined in the text. Note the appearance of a pronounced peak at $\simeq-0.5 \mathrm{eV}$ in the $\mathrm{O} 4 \mathrm{PDOS}$ of the disordered structure.

with the value of $0.46 \mathrm{eV}$ obtained from Monte Carlo simulations [23, 24, 25]. Thus, the driving force of disorder in YSCO does not arise from total energy differences. We then evaluate the entropy contribution made of the electronic, configurational and phononic terms. The latter term can not be easily calculated, especially in disordered structures. Though, in first approximation, we may neglect this term considering that the lattice dynamics in the ordered and disordered phases should not differ significantly from YBCO to YSCO. This is indeed a reasonable assumption considering that the relevant quantity entering the entropy expression is the phononic DOS and previous neutron scattering [26] and specific heat [27] studies show that this quantity does not change appreciably in YBCO upon substituting $\mathrm{Y}$ with a heavier rare earth element. This result is not surprising considering that the isovalent chemical substitution of only one of the 13 atoms forming the unit cell will affect only a limited number of phonon modes. A similar situation should then occur in the present case of Sr/Ba substitution.

We then estimate the remaining two terms. As for the electronic term, we recall that, in YBCO, the disruption of the chain-related $\pi$-antibonding bands reduce $N\left(E_{F}\right)$ and, consequently, the electronic entropy. We conclude that, in YBCO, the electronic entropy tends to stabilise the ordered phase with long chains. The opposite conclusion is drawn for YSCO for two reasons. 1) The $\pi$ band is located below $E_{F}$, independent of the ordering; 2) the disorder lowers a chain-related $d_{z^{2}-y^{2}} \sigma$-antibonding band, located above $E_{F}$ in the ordered phase. At the $S^{\prime}$-point, this band even crosses $E_{F}$, thus enhancing $N\left(E_{F}\right)$ and the entropic term favorable to the disordered phase. 
Quantitatively, we estimate the above differences by computing the difference $\Delta S \equiv S_{d i s}-S_{\text {ord }}$ between ordered and disordered phases using the usual expression

$$
S_{e l}^{(d i s, \text { ord })}=-k_{B} \int N^{(d i s, o r d)}(\varepsilon)[f(\varepsilon) \ln f(\varepsilon)+(1-f(\varepsilon)) \ln (1-f(\varepsilon)] \gamma d \varepsilon
$$

where $\gamma \equiv m^{*} / m$ is the effective mass enhancement factor for the electron, $k_{B}$ is the Boltzmann constant, $f(\varepsilon)$ is the Fermi-Dirac distribution function and the density of states $N\left(E_{F}\right)$ is obtained from accurate fits of the energy bands. The above expression must be evaluated at the temperature $T=1323 \mathrm{~K}$ at which the YSCO samples are synthesised and subsequently quenched down to room temperature [4]. For YBCO, we obtain a negative entropic contribution to the free energy $T \Delta S_{e l} \approx-0.07 \gamma \mathrm{eV} / \mathrm{supercell}$. By using the experimental $\gamma=5$ value reported for fully doped YBCO [28], we obtain a contribution of comparable magnitude, but with opposite sign, with respect to the positive difference of configurational entropy $T \Delta S_{\text {conf }}=2 k_{B} T \ln (2)=0.158 \mathrm{eV}$. In conclusion, in YBCO, the algebraic sum of both contributions to $T \Delta S$ is estimated to be smaller than the total energy difference. This conclusion is in agreement with the stability of the orthorhombic phase of YBCO found experimentally. On the contrary, in YSCO, we estimate a positive electronic contribution $T \Delta S_{e l} \approx 0.04 \gamma \mathrm{eV}$. Consequently, the sum of configurational and electronic contributions to the entropic term TS is positive and sizeable. By assuming again $\gamma=5$, we obtain $T \Delta S_{e l+c o n f} \approx 0.36 \mathrm{eV}$. The latter value clearly indicates that the disorder is more favored in YSCO than in YBCO but the corresponding free-energy gain is still smaller than the relevant energy difference. Therefore our calculations would fully account for the disorder in stoichiometric YSCO only in the case of higher $\gamma \approx 8-10$ values, as reported in Ref.[29, 30], or if the phononic contribution would play an important role.

A key to elucidate this point may be provided by a high-resolution transmission electron microscopy study on both over- $(x=1.3)$ and under-stoichiometric $(x=0.9)$ compounds [6]. This study shows that the former compounds, with 0.3 excess oxygens per unit cell with respect to the stoichiometric phase $[3,4]$, exhibit no trace of oxygen ordering in the local scale. On the contrary, the latter compounds mainly consist of a modulated phase with $4 a$-long $\mathrm{CuO}$ chain fragments. We conclude that oxygen overdoping is likely to favor the disordered phase. In turn, $T_{c}$ increases by only 4-5

$\mathrm{K}$ upon reducing $x$ from 1.3 to 0.9 , which indicates that the as-prepared samples are overdoped and that the maximum $T_{c}$ is intrinsically smaller in YSCO than in YBCO.

\section{Conclusions}

In conclusion, we have studied the effects of oxygen disorder on the electronic structure of stoichiometric $\mathrm{YSr}_{2} \mathrm{Cu}_{3} \mathrm{O}_{6+x}$ (i.e. for $x=1$ ) by calculating the $a b$ initio band structure of two tetragonal supercells simulating disordered $\mathrm{CuO}$ chains and of an hypothetical orthorhombic structure with ordered $\mathrm{CuO}$ chains, isostructural to that of stoichiometric $\mathrm{YBa}_{2} \mathrm{Cu}_{3} \mathrm{O}_{6+x}$. For comparison, we carried out the same calculations on the latter phase as well. In agreement with experiments, we find that the orthorhombic ordered 
phase, where $\mathrm{CuO}$ chains are formed along the $b$-axis direction, is thermodynamically favored in $\mathrm{YBa}_{2} \mathrm{Cu}_{3} \mathrm{O}_{6+x}$. In $\mathrm{YSr}_{2} \mathrm{Cu}_{3} \mathrm{O}_{6+x}$, we find an incipient oxygen disorder that may be stabilised by oxygen overdoping, as suggested by the experimental evidence of average tetragonal symmetry and by the absence of any oxygen ordering in the local scale reported for samples with $x=1.3$. This phenomenon provides a hint to explain the $30 \mathrm{~K}$ reduction of $T_{c}$ in YSCO with respect to YBCO and raises the question whether a similar situation may contribute to the large $T_{c}$ variations observed in cuprates.

\section{Acknowledgments}

The authors acknowledge fruitful discussions with E. Gilioli, F. Licci, M. Marezio and P.G. Radaelli.

\section{References}

[1] Bednorz J G and Müller K A 1986 Zeitschrift für Physik B Condensed Matter 64 189-193 ISSN 1431-584X URL https://doi.org/10.1007/BF01303701

[2] Okai B 1990 Japanese Journal of Applied Physics 29 L2193 URL http://stacks . iop.org/1347-4065/29/i=12A/a=L2193

[3] Cao Y, Hudson T L, Wang Y S, Xu S H, Xue Y Y and Chu C W 1998 Phys. Rev. B 58(17) 11201-11203 URL https://link.aps.org/doi/10.1103/PhysRevB.58.11201

[4] Gilioli E, Radaelli P, Gauzzi A, Licci F and Marezio M 2000 Physica C: Superconductivity 341-348 $605-606$ ISSN 0921-4534 materials and Mechanisms of Superconductivity High Temperature Superconductors VI URL http://www.sciencedirect.com/science/article/pii/S0921453400006109

[5] Gauzzi A, Gilioli E, Licci F, Marezio M, Massidda S, Bernardini F, Continenza A and Radaelli P G 2000 Proc. SPIE 40584058 - 4058

[6] Lebedev O I, Van Tendeloo G, Licci F, Gilioli E, Gauzzi A, Prodi A and Marezio M 2002 Phys. Rev. B 66(13) 132510 URL https://link.aps.org/doi/10.1103/PhysRevB.66.132510

[7] Prodi A, Gauzzi A, Gilioli E, Licci F, Marezio M, Bolzoni F, Allodi G, De Renzi R, Bernardini F, Massidda S, Profeta G, Continenza A and Radaelli P G 2003 International Journal of Modern Physics B 17 873-878 (Preprint https://doi.org/10.1142/S0217979203016753) URL https://doi.org/10.1142/S0217979203016753

[8] Jorgensen J, Pei S, Lightfoor P, Shi H, Paulikas A and Veal B 1990 Physica C: $\quad$ Superconductivity $\mathbf{1 6 7} \quad 571 \quad-\quad 578$ ISSN $0921-4534$ URL http://www.sciencedirect.com/science/article/pii/0921453490906766

[9] Poulsen H F, Andersen N H, Andersen J V, Bohr H and Mouritsen O G 1991 Phys. Rev. Lett. 66(4) 465-468 URL https://link.aps.org/doi/10.1103/PhysRevLett.66.465

[10] Burdett J K 1992 Physica C: Superconductivity 191282 - 291 ISSN 0921-4534 URL http://www.sciencedirect.com/science/article/pii/0921453492906605

[11] Jansen H J F and Freeman A J 1984 Phys. Rev. B 30(2) 561-569 URL https://link.aps.org/doi/10.1103/PhysRevB.30.561

[12] Wimmer E, Krakauer H, Weinert M and Freeman A J 1981 Phys. Rev. B 24(2) 864-875 URL https://link.aps.org/doi/10.1103/PhysRevB.24.864

[13] Monkhorst H J and Pack J D 1976 Phys. Rev. B 13(12) 5188-5192 URL https://link.aps.org/doi/10.1103/PhysRevB.13.5188

[14] Kresse G and Hafner J 1993 Phys. Rev. $B \quad$ 47(1) 558-561 URL https://link.aps.org/doi/10.1103/PhysRevB.47.558 
[15] Kresse G and Furthmüller J 1996 Phys. Rev. B 54(16) 11169-11186 URL https://link.aps.org/doi/10.1103/PhysRevB.54.11169

[16] Vanderbilt D $1990 \quad$ Phys. Rev. B $41(11) \quad 7892-7895 \quad$ URL https://link.aps.org/doi/10.1103/PhysRevB.41.7892

[17] Ceperley D M and Alder B J 1980 Phys. Rev. Lett. 45(7) 566-569 URL https://link.aps.org/doi/10.1103/PhysRevLett.45.566

[18] Perdew J P and Zunger A 1981 Phys. Rev. B 23(10) 5048-5079 URL https://link.aps.org/doi/10.1103/PhysRevB.23.5048

[19] Long R and Prezhdo O V 2017 The Journal of Physical Chemistry Letters 8 193-198 URL https://doi.org/10.1021/acs.jpclett.6b02713

[20] Capponi J J, Chaillout C, Hewat A W, Lejay P, Marezio M, Nguyen N, Raveau B, Soubeyroux J L, Tholence J L and Tournier R 1987 EPL (Europhysics Letters) $31301 \mathrm{URL}$ http://stacks.iop.org/0295-5075/3/i=12/a=009

[21] Yu J, Massidda S, Freeman A and Koeling D 1987 Physics Letters A 122203 - 208 ISSN 0375-9601 URL http://www.sciencedirect.com/science/article/pii/0375960187908073

[22] Simon A, Köhler J, Borrmann H, Gegenheimer B and Kremer R 1988 Journal of Solid State Chemistry $\mathbf{7 7} \quad 200 \quad-\quad 203$ ISSN $0022-4596$ URL http://www.sciencedirect.com/science/article/pii/0022459688901107

[23] Mønster D, Lindgård P A and Andersen N H 1999 Phys. Rev. B 60(1) 110-113 URL https://link.aps.org/doi/10.1103/PhysRevB.60.110

[24] Mannstaedt S and Pawley G S 2000 Phys. Rev. B 62(6) 4169-4174 URL https://link.aps.org/doi/10.1103/PhysRevB.62.4169

[25] Mønster D, Lindgård P A and Andersen N H 2001 Phys. Rev. B 64(22) 224520 URL https://link.aps.org/doi/10.1103/PhysRevB.64.224520

[26] Renker B, Gompf F, Gering E, Roth G, Reichardt W, Ewert D and Rietschel H 1988 Physica $C$ : Superconductivity 153-155 $272-273$ ISSN 0921-4534 URL http://www.sciencedirect.com/science/article/pii/0921453488905886

[27] Meulen H V D, Franse J, Tarnawski Z, Kadowaki K, Klaasse J and Menovsky A 1988 Physica C: Superconductivity 15265 - 71 ISSN 0921-4534 URL http://www.sciencedirect.com/science/article/pii/0921453488900731

[28] Fiory A T, Hebard A F, Eick R H, Mankiewich P M, Howard R E and O'Malley M L 1990 Phys. Rev. Lett. 65(27) 3441-3444 URL https://link.aps.org/doi/10.1103/PhysRevLett.65.3441

[29] Yeh N C and Tsuei C $\mathrm{C} 1989$ Phys. Rev. B 39 39(13) 9708-9711 URL https://link.aps.org/doi/10.1103/PhysRevB.39.9708

[30] Hopfengärtner R, Leghissa M, Kreiselmeyer G, Holzapfel B, Schmitt P and Saemann-Ischenko G 1993 Phys. Rev. B $\mathbf{4 7 ( 1 0 )}$ 5992-6003 URL https://link.aps.org/doi/10.1103/PhysRevB.47.5992 